\title{
Multiple centrosomes: together they stand, divided they fall
}

\author{
Fanni Gergely ${ }^{1,4}$ and Renata Basto ${ }^{2,3}$ \\ ${ }^{1}$ Cancer Research UK, Cambridge Research Institute, Li Ka Shing Centre, Department of Oncology, University \\ of Cambridge, Cambridge CB2 ORE, United Kingdom; ${ }^{2}$ UMR 144, CNRS, Institut Curie, Paris Cedex 05, France
}

Cells with extra centrosomes rely entirely on centrosome clustering mechanisms to assemble a bipolar spindle and to divide in a bipolar fashion. To identify the pathways involved in suppression of multipolarity, Kwon, Godinho, and colleagues (pp. 2189-2203) have set up a genome-wide screen in Drosophila S2 cells. Surprisingly, they found that efficient clustering requires a large number of proteins associated with a variety of cellular functions.

The majority of cells in our body contain either one or two centrosomes, depending on their position within the cell cycle. A G1 cell contains a single centrosome composed of two centrioles. During $S$ phase, two new (daughter) centrioles assemble in the close proximity of preexisting (mother) centrioles, therefore generating two centrosomes that remain together until the end of G2. As mitosis is initiated, the two centrosomes move apart to form the poles of a bipolar mitotic spindle (Doxsey et al. 2005; Azimzadeh and Bornens 2007; Bettencourt-Dias and Glover 2007).

Centrosome number is maintained by the tight control of the centrosome replication cycle. Although in the past few years, our understanding of the mechanisms that regulate centrosome replication have improved considerably, how cells manage to restrict the formation of daughter centrioles to only two per cell cycle is still unclear (Tsou and Stearns 2006; Azimzadeh and Bornens 2007; Bettencourt-Dias and Glover 2007; Nigg 2007).

Increased centrosome number, or centrosome amplification, is a common characteristic of several human tumors. The general perception is that elevated centrosome numbers are associated with multipolar mitosis and consequently with abnormal cell division ( $\mathrm{D}^{\prime}$ Assoro et al. 2002a,b; Boveri 2008). The cause for centrosome amplification in tumors is not known, but, in principle, the presence of multiple centrosomes could result from defects in the control of the centrosome replication cycle, failure in cytokinesis, or, alternatively, cell fusion.

[Keywords: Centrosomes; mitosis; actin; adhesion; cancer; cell cycle] Correspondence.

${ }^{3}$ E-MAIL renata.bastro@curie.fr; FAX 33-1-56-24-6377.

${ }^{4}$ E-MAIL Fanni.Gergely@cancer.org.uk; FAX 44-1223-404-573.

Article is online at http://www.genesdev.org/cgi/doi/10.1101/gad.1715208.
The exact contribution of extra centrosomes to tumor initiation is still a matter for discussion. The coexistence, in certain tumors and in tumor-derived cell lines, of multiple centrosomes together with high levels of aneuploidy suggests a positive correlation between centrosome amplification and chromosome instability (Lingle et al. 2002). However, the presence of extra centrosomes could also disrupt cell polarity and consequently interfere with normal cell behavior and tissue architecture. In vivo, centrosome amplification has been recently shown to drive tumorigenesis in flies (Basto et al. 2008), but it is too early to say if this holds true in other model organisms.

In theory, the presence of extra centrosomes within tumor cells could also prove to be deleterious. Multipolar mitosis, for instance, may generate sufficiently high levels of aneuploidy to pose a challenge for cell viability. Interestingly, several cancer cell lines seem to overcome this problem by clustering their extra centrosomes at the two poles of the spindle during mitosis, thus ensuring bipolar chromosome segregation (Ring et al. 1982; Quintyne et al. 2005; Saunders 2005).

Although centrosome clustering occurs both in vivo (Murphy 2003; Basto et al. 2008) and in tissue culture cells (Ring et al. 1982; Quintyne et al. 2005), the molecular mechanism responsible is poorly understood. Previous work has implicated the minus-end-directed motor Dynein and the spindle pole-associated protein NuMA in this process, but further proteins and pathways are also likely to be involved (Quintyne et al. 2005; Saunders 2005).

To investigate the mechanisms that regulate centrosome clustering, Kwon and Godinho from the Pellman laboratory have set up a clever screen in Drosophila S2 culture cells (Kwon et al. 2008). S2 cells are ideal for such a screen for two major reasons. First, more than $50 \%$ of these cells contain multiple centrosomes. Second, S2 cells are extremely efficient at clustering extra centrosomes, since $95 \%$ of the cells assemble a bipolar mitotic spindle (Fig. 1A). The investigators screened a total of 23,172 genes, which correspond to the majority of the Drosophila genome (99\%), by RNAi. Careful visual analysis of $\sim 96,000$ images identified 133 genes with roles in centrosome clustering implicated in a variety of cellular functions. 


\section{Time is everything}

A group of genes identified by this screen are members of the Spindle Assembly Checkpoint (SAC) required for controlling the timing of anaphase onset. The SAC is a surveillance mechanism that monitors the kinetochoremicrotubule (MT) attachment status of all chromosomes within a cell (Musacchio and Salmon 2007). This checkpoint delays anaphase onset by maintaining the Anaphase-Promoting Complex/Cyclosome (APC/C, an E3 ubiquitin ligase) in an inactive form (Acquaviva and Pines 2006). When the SAC is absent in cells containing extra centrosomes, multipolarity is maintained throughout mitosis and chromosomes are segregated in a multipolar fashion. Interestingly, comparing the time elapsed between nuclear envelope breakdown (NEBD) and anaphase onset in S2 cells with two centrosomes or more revealed a positive correlation between the presence of extra centrosome and the length of mitosis. To investigate the role of SAC in promoting centrosome clustering, the investigators blocked anaphase progression (in SAC- depleted cells) with a proteasome inhibitor. Interestingly, suppression of multipolarity was partially rescued, which suggests that it is not the presence of extra centrosomes that is being monitored by the SAC. Instead, kinetochore-MT attachment, in these multipolar arrangements, might maintain the SAC in an active state, which in turn provides the cell with sufficient time to cluster supernumerary centrosomes and to assemble a bipolar spindle (Fig. 1B). The dependence of centrosome clustering and bipolar spindle formation on a functional SAC also has been described recently in other models (Basto et al. 2008; Yang et al. 2008).

\section{Motors and forces: a fine balancing act}

Another class of proteins identified by this screen are MT-binding proteins with roles in spindle pole focusing. One of the strongest hits identified was a molecular motor, the minus-end kinesin Ncd or HSET, which belongs to the Kinesin-14 family. This result is particularly in-
Figure 1. Genome-wide screen in cells with supernumerary centrosomes identifies three major pathways required for centrosome clustering. $(A)$ In interphase, several centrosomes (green dots) can be detected, randomly distributed in the cytoplasm of S2 cells. Cells were incubated with dsRNA for $4 \mathrm{~d}$ and stained for DNA, MTs, and centrosomes. Using high throughput automated microscopy to acquire -96000 images, Kwon et al. (2008) quantified the percentage of multipolar spindles for each gene knock down. A secondary screen confirmed that 133 genes are required to cluster multiple centrosomes and to assemble a bipolar mitotic spindle. $(B)$ Mechanisms required for centrosome clustering. (1) The SAC. The presence of multipolar spindles is likely to perturb kinetochore-MT attachment, and cells arrest in a prometaphase like state with an active checkpoint that inhibits the APC/C. With enough time, centrosomes cluster and a bipolar spindle is assembled. Kinetochore-MT attachment is then likely to satisfy the checkpoint, and the APC/C can now be activated to trigger anaphase and mitotic exit. (2) MAPs as Ncd/HSET facilitate centrosome clustering. As cells enter in mitosis, Ncd is required to block centrosome movement away from the poles (toward the cortex). These proteins probably contribute to generate spindle forces that maintain the extra centrosomes together throughout mitosis. (3) Cell adhesion, the actin, and MT cytoskeleton cooperate to promote clustering. Cortical landmarks that form on the proximal sides of interphase adhesion sites recruit a large amount of factors that organize the actin and microtubule cytoskeleton. In a cell with multiple centrosomes, these landmarks generate a cortical force that exerts a pulling force on stable astral microtubules. Whether landmarks and cortical forces promote or suppress clustering depends on the arrangements of landmarks (e.g., two on opposite sides of the cell would favor clustering, whereas three or four arranged in a symmetric fashion would impede clustering).

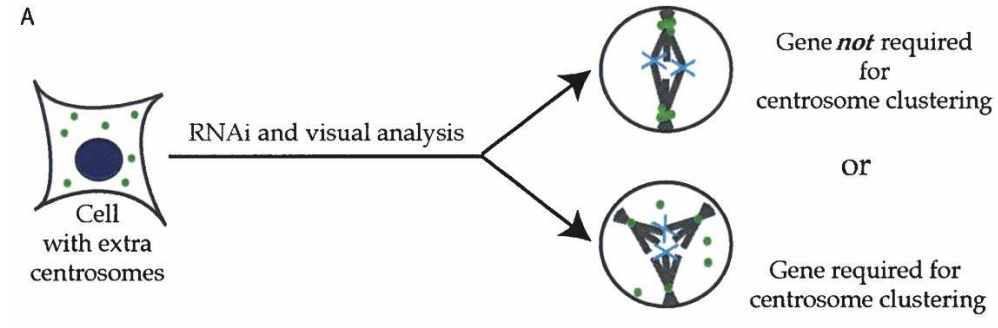

B

1- The Spindle assembly checkpoint

Prometaphase arrest Mitotic progression
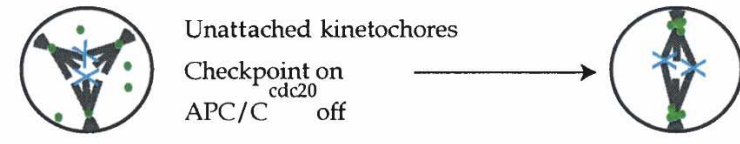

All kinetochores correctly attached Checkpoint off $\mathrm{APC} / \mathrm{C}^{\mathrm{cdc} 20}$ on

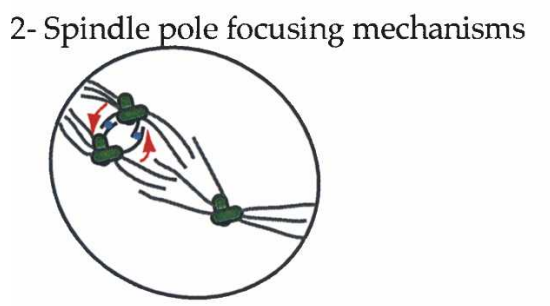

3- Cell adhesion-based centrosome clustering

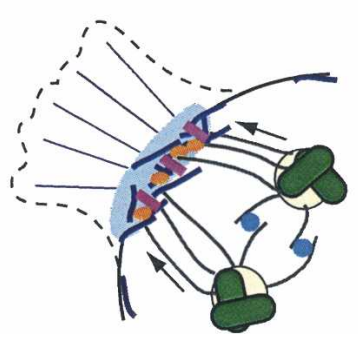




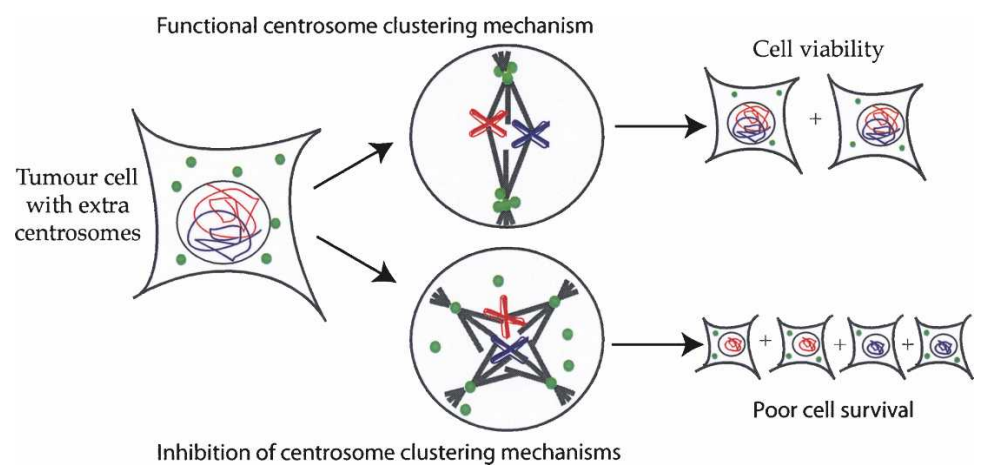

Figure 2. Centrosome amplification and cancer therapy. The presence, within a tumor, of a high portion of cells with extra centrosomes that rely on centrosome clustering mechanisms to continue to divide and thus to proliferate might be of therapeutic interest. In principle, inhibition of centrosome clustering mechanisms may result in multipolar spindle assembly and abnormal cell division and consequently in the production of low-viability progeny. As shown by Kwon et al. (2008), HSET is essential for the survival of cancer cells with extra centrosomes but is dispensable for mitosis in cells with only two centrosomes. teresting, since Drosophila ncd mutants are viable but female sterile (Endow and Komma 1998). Previous work has shown that in Drosophila somatic cells that contain normal centrosome number Ncd is dispensable. The activity of this motor is, however, essential to focus the MT-minus ends of the acentrosomal female meiotic spindle (Skold et al. 2005). In Drosophila, like in the majority of animal species, female meiotic spindles do not contain centrosomes. In this case, MT nucleation entirely depends on the chromatin-mediated pathway and on the role of additional molecular motors to focus MTs at the spindle poles (Heald et al. 1996, 1997; Skold et al. 2005; Barr and Gergely 2007).

Live analysis of ncd-depleted S2 cells revealed increased centrosome mobility toward the cell cortex, away from the spindle. Thus, Ncd activity is important for both suppressing centrosome movement and constraining its position, most likely by balancing cortical forces acting on the centrosomes (Fig. 1B). Interestingly, while certain MT-associated proteins (MAPs) such as Asp, Myo10A, Clip-190, and Tankyrase are among the candidates required for suppression of multipolarity, other MAPs with well-known roles in mitosis such as EB1 (Vaughan 2005), Msps/ChTog (Cullen et al. 1999; Gergely et al. 2003), or D-TACC (Gergely et al. 2000; Raff 2002) have either been identified as a weak hit or not identified at all. This suggests a requirement for a specific small subset of MAPs in centrosome clustering.

\section{Centrosome clustering and spindle positioning: two sides of the same coin?}

As described above, cell autonomous factors such as the SAC and intrinsic spindle forces can facilitate the clustering of centrosomes. Environmental cues, however, seem to play just as an important role according to the RNAi screen by Kwon et al. (2008). In fact, $>30 \%$ of candidate proteins, with known functions, identified by the screen are involved in the regulation of the actin cytoskeleton, cell polarity, adhesion, or cortical contractility. A closer look at the list of factors within these categories reveals a striking overlap with a group of proteins involved in cell adhesion-dependent spindle positioning (Thery et al. 2005; Toyoshima and Nishida 2007). Hence, cells with extra centrosomes seem to have "hi- jacked" the spindle positioning mechanism to promote their survival. As detailed below, this clustering mechanism relies on adhesion- and actin-dependent generation of heterogeneous cortical forces that organize astral MTs and thereby guide centrosome movement.

While the actin cytoskeleton has a well-established role in cell shape, motility, cytokinesis, and spindle positioning, the current study is the first to suggest that it promotes centrosome clustering in cells with extra centrosomes (Kwon et al. 2008). Disrupting the actin cytoskeleton by the depolymerizing agent Latrunculin A (Lat A) causes a twofold increase in multipolar mitoses in both S2 and mammalian cells. Lat A treatment of Ncd/ HSET-depleted cells revealed a synergistic effect on multipolarity, whereas Lat A treatment did not further increase multipolarity in cells depleted of factors involved in cell adhesion and contractility. Therefore, centrosome clustering by Ncd/HSET occurs independently of the actin cytoskeleton, while cell adhesion and contractility factors facilitate clustering in an actin-dependent manner (Kwon et al. 2008). So, how can cell adhesion and cortical contractility link the actin cytoskeleton to centrosome clustering?

Most adherent cell types in tissue culture adopt a round morphology during mitosis, a shape that promotes the stability of mitotic spindles (Kunda et al. 2008). Cell rounding is accompanied by the detachment of the plasma membrane from the substrate as a result of actomyosin-dependent cortical contractions. Stable anchoring sites, however, resist these forces, allowing the formation of actin-based retraction fibers (RFs) that persist throughout mitosis (Mitchison 1992). Cell-matrix adhesion molecules are paramount in the establishment of these RFs (Thery et al. 2005). The FERM-domain protein Fit1A identified in the screen is a good candidate for such a role, since its mammalian homolog, Mig-2, is required for cell spreading possibly by providing a link between integrins and the actin cytoskeleton (Tu et al. 2003; Shi et al. 2007). To directly address the connection between cell adhesion, RF assembly and clustering, Kwon et al. (2008) performed a series of elegant experiments using time-lapse microscopy methods to follow live human cells on fibronectin-coated surfaces. The investigators noticed that extra centrosome-containing human breast cancer cells with elongated interphase morphology as- 
semble multipolar spindles less frequently than cells with round morphology. Therefore, interphase cell shape exerts a strong influence on the efficiency of centrosome clustering in the subsequent mitosis. In addition, bipolar spindle formation is clearly favored when cells are grown on fibronectin micropatterns with a two-pole symmetry (I or $\mathrm{H}$ shape). Patterns such as $\mathrm{Y}$ and $\mathrm{O}$ are sufficient to cause a threefold increase in the frequency of multipolar spindles. These results cumulatively suggest that the physical environment of an interphase cell has a major impact on how effectively extra centrosomes can cluster during spindle assembly. But, how can the distribution of RFs influence centrosome clustering?

The ability of RFs to modulate the actin and MT cytoskeletons plays an important role in spindle positioning (Thery et al. 2005, 2007; Toyoshima and Nishida 2007), and it may also be exploited in centrosome clustering (Kwon et al. 2008). RFs generate a heterogeneous cortex by selectively accumulating factors such as members of the ezrin/radixin/moesin (ERM) family, hence turning cell adhesion patterns into cortical landmarks (Bretscher et al. 2002; Thery and Bornens 2006). These landmarks are important for cortical contractility, MT organization, and stable spindle positioning (Carreno et al. 2008; Kunda et al. 2008). A positive relation was demonstrated between cortical contractility and centrosome clustering in drug-based assays that modulate contractility; impaired contractility reduced clustering, whereas increased contractility was associated with improved clustering. A direct role for contractility in centrosome clustering was also suggested by the presence of Myo10A-a member of the atypical myosin familyamong the high-scoring candidate proteins. Importantly, drug-induced contractility restored normal centrosome clustering in Myo10A-depleted cells, indicating that the primary role of Myo10A in clustering is to facilitate cortical contractions (Kwon et al. 2008). The function of contractility in centrosome clustering is evolutionarily conserved, as depletion of Myo-10, a related atypical myosin in mammals with an established role in spindle positioning (Toyoshima and Nishida 2007), suppressed clustering in human cells with supernumerary centrosomes.

Astral MTs seem to play a crucial role in centrosome clustering since their selective depolymerization increased the frequency of multipolar spindles (Kwon et al. 2008). The RFs could locally influence astral MT stability via the recruitment of stabilizing proteins. Stable astral MTs establish a positive feedback loop by transporting further stabilizing factors from the spindle poles to the RF (Thery and Bornens 2006), leading to the enrichment of MT plus-end stabilizing complexes such as CLIP-170/IQGAP/Rac1/cdc42 (Fukata et al. 2002) at these sites. This complex may play a role in centrosome clustering, since the Drosophila CLIP-170 homolog, CLIP-190, has been identified as a high-scoring candidate protein in the siRNA screen. The similarities between the molecular pathways involved in spindle positioning and centrosome clustering are so intriguing that it might be worthwhile testing certain candidates identified in the Kwon and Godinho screen for their potential role in spindle positioning (Kwon et al. 2008).

Interestingly, polarity proteins such as Crumbs (Tepass et al. 1990), Cornetto (Bulgheresi et al. 2001), and PAR-1 (Shulman et al. 2000; Doerflinger et al. 2003) have also been isolated as candidate proteins in centrosome clustering. Besides the role of PAR-1 in epithelial polarity, MT cytoskeleton polarization, and asymmetric cell division (Margolis and Borg 2005), there is also evidence for the involvement of PAR-1 in cell-matrix adhesion (Kiger et al. 2003). These polarity proteins must influence centrosome clustering through the actin cytoskeleton, since depleting them in the presence of Lat A does not cause a synergistic increase in multipolar spindles (Kwon et al. 2008).

While it is clear that actin-driven forces contribute to the clustering of extra centrosomes, the precise molecular mechanisms involved remain to be established. Thery et al. (2007) have suggested recently that heterogeneous cortical forces exert a pulling force on astral MTs, hence producing a torque on the mitotic spindle, which rotates it until stable spindle orientation is achieved. It is possible that centrosome clustering occurs via a similar mechanism. It will be crucial to determine the identity of the major players involved in the generation of these forces. Since such factors may be among the $43 \%$ of candidate proteins that have no known function, screening this panel in the presence of Lat A could be a quick way to identify missing molecular links.

The Kwon and Godinho screen may also have broader implications on environment-induced centrosome positioning within cells (Kwon et al. 2008). For instance, centrosome movement during epithelial polarization, membrane targeting of the centrosome in preparation to form the primary cilium, and centrosome positioning during the process of wound healing could all require some extent of actin remodeling. It is therefore feasible that a universal pathway exists to link the remodeling of the cortical actin cytoskeleton to MT organization and centrosome positioning in cells.

\section{Centrosome clustering and cancer therapeutics}

Centrosome amplification is a common occurrence in a variety of human cancers. As discussed above, the success of cell division in the presence of supernumerary centrosomes depends entirely on efficient centrosome clustering mechanisms that promote bipolar spindle assembly and accurate distribution of sister chromatids during anaphase. This could make the clustering ability of a cancer cell with excess number of centrosomes its Achilles heel (Fig. 2; Kwon et al. 2008). The finding that centrosome clustering pathways are dispensable in cells containing normal centrosome number establishes the process of centrosome clustering as an attractive, tumor cell-specific therapeutic target. Indeed, as demonstrated by Kwon et al. (2008), HSET depletion blocks centrosome clustering and promotes multipolar divisions and hence abnormal chromosome segregation or aneuploidy. Most importantly, HSET depletion selectively kills cells with 
extra centrosomes without affecting the viability of cells that contain normal centrosome numbers. Consequently, based on the in vitro data, the incidence of toxicity may be markedly lower with a hypothetical HSET inhibitor than with other mitosis-blocking agents currently in clinical use. HSET is therefore an exciting new therapeutic target, and being a kinesin, it is also a great candidate for small molecule inhibitor-based approaches. In summary, inhibiting centrosome clustering could turn out to be a very powerful way to shrink tumors, in particular those with a high penetrance of centrosome amplification.

It is worth a mention, however, that aneuploidy does not only act as a tumor suppressor (Sotillo et al. 2007; Weaver et al. 2007) but can also behave as a tumor initiator at least in certain tissues of aged animals (Weaver et al. 2007). It will be critical, therefore, to ensure that a potential HSET inhibitor does not generate excessive amounts of low degree aneuploidy within tumors.

It is important to recall that centrosome clustering can occur via at least two distinct mechanisms: One is intrinsic to the spindle, whereas the other relies on actingenerated forces in response to environmental cues. These two pathways cooperate under tissue culture conditions to bring about efficient clustering, but in vivo cells may utilize one or the other pathway depending on their shape and environment. It would be particularly interesting to see which pathway prevails in cells with multiple centrosomes when grown in a three-dimensional tissue culture system or in in vivo vertebrate models. A preferential use of clustering pathways is already apparent in two-dimensional culture conditions where the small, round, and nonadherent neuroblastoma cell line relies on HSET almost entirely for centrosome clustering, whereas adherent cells seem to tolerate the absence of HSET better (Kwon et al. 2008). Tumor cells with supernumerary centrosomes will therefore select clustering pathways that afford the best chance for survival within their particular environment.

\section{Acknowledgments}

F.G. is a Royal Society University Research Fellow. Research in F.G.'s laboratory is supported by Cancer Research UK. Research in R.B.'s group is supported by the CNRS (UMR 144), the FRM, and the Institut Curie.

\section{References}

Acquaviva, C. and Pines, J. 2006. The anaphase-promoting complex/cyclosome: APC/C. J. Cell Sci. 119: 2401-2404.

Azimzadeh, J. and Bornens, M. 2007. Structure and duplication of the centrosome. J. Cell Sci. 120: 2139-2142.

Barr, A.R. and Gergely, F. 2007. Aurora-A: The maker and breaker of spindle poles. J. Cell Sci. 120: 2987-2996.

Basto, R., Brunk, K., Vinadogrova, T., Peel, N., Franz, A., Khodjakov, A., and Raff, J.W. 2008. Centrosome amplification can initiate tumorigenesis in flies. Cell 133: 1032-1042.

Bettencourt-Dias, M. and Glover, D.M. 2007. Centrosome biogenesis and function: Centrosomics brings new understanding. Nat. Rev. Mol. Cell Biol. 8: 451-463.
Boveri, T. 2008. Concerning the origin of malignant tumours by Theodor Boveri. Translated and annotated by Henry Harris. J. Cell Sci. 121(Suppl. 1): 1-84. doi: 10.1242/10.1242/ jcs.025742.

Bretscher, A., Edwards, K., and Fehon, R.G. 2002. ERM proteins and merlin: Integrators at the cell cortex. Nat. Rev. Mol. Cell Biol. 3: 586-599.

Bulgheresi, S., Kleiner, E., and Knoblich, J.A. 2001. Inscuteabledependent apical localization of the microtubule-binding protein Cornetto suggests a role in asymmetric cell division. J. Cell Sci. 114: 3655-3662.

Carreno, S., Kouranti, I., Glusman, E.S., Fuller, M.T., Echard, A., and Payre, F. 2008. Moesin and its activating kinase Slik are required for cortical stability and microtubule organization in mitotic cells. J. Cell Biol. 180: 739-746.

Cullen, C.F., Deak, P., Glover, D.M., and Ohkura, H. 1999. mini spindles: A gene encoding a conserved microtubule-associated protein required for the integrity of the mitotic spindle in Drosophila. J. Cell Biol. 146: 1005-1018.

D'Assoro, A.B., Barrett, S.L., Folk, C., Negron, V.C., Boeneman, K., Busby, R., Whitehead, C., Stivala, F., Lingle, W.L., and Salisbury, J.L. 2002a. Amplified centrosomes in breast cancer: A potential indicator of tumor aggressiveness. Breast Cancer Res. Treat. 75: 25-34.

D'Assoro, A.B., Lingle, W.L., and Salisbury, J.L. 2002b. Centrosome amplification and the development of cancer. Oncogene 21: 6146-6153.

Doerflinger, H., Benton, R., Shulman, J.M., and St Johnston, D. 2003. The role of PAR-1 in regulating the polarised microtubule cytoskeleton in the Drosophila follicular epithelium. Development 130: 3965-3975.

Doxsey, S., Zimmerman, W., and Mikule, K. 2005. Centrosome control of the cell cycle. Trends Cell Biol. 15: 303-311.

Endow, S.A. and Komma, D.J. 1998. Assembly and dynamics of an anastral:astral spindle: The meiosis II spindle of Drosophila oocytes. J. Cell Sci. 111: 2487-2495.

Fukata, M., Watanabe, T., Noritake, J., Nakagawa, M., Yamaga, M., Kuroda, S., Matsuura, Y., Iwamatsu, A., Perez, F., and Kaibuchi, K. 2002. Rac1 and Cdc42 capture microtubules through IQGAP1 and CLIP-170. Cell 109: 873-885.

Gergely, F., Kidd, D., Jeffers, K., Wakefield, J.G., and Raff, J.W. 2000. D-TACC: A novel centrosomal protein required for normal spindle function in the early Drosophila embryo. EMBO J. 19: 241-252.

Gergely, F., Draviam, V.M., and Raff, J.W. 2003. The ch-TOG/ XMAP215 protein is essential for spindle pole organization in human somatic cells. Genes \& Dev. 17: 336-341.

Heald, R., Tournebize, R., Blank, T., Sandaltzopoulos, R., Becker, P., Hyman, A., and Karsenti, E. 1996. Self-organization of microtubules into bipolar spindles around artificial chromosomes in Xenopus egg extracts. Nature 382: 420-425.

Heald, R., Tournebize, R., Habermann, A., Karsenti, E., and Hyman, A. 1997. Spindle assembly in Xenopus egg extracts: Respective roles of centrosomes and microtubule self-organization. J. Cell Biol. 138: 615-628.

Kiger, A.A., Baum, B., Jones, S., Jones, M.R., Coulson, A., Echeverri, C., and Perrimon, N. 2003. A functional genomic analysis of cell morphology using RNA interference. J. Biol. 2: 27. doi: 10.1186/1475-4924-2-27.

Kunda, P., Pelling, A.E., Liu, T., and Baum, B. 2008. Moesin controls cortical rigidity, cell rounding, and spindle morphogenesis during mitosis. Curr. Biol. 18: 91-101.

Kwon, M., Godinho, S.A., Chandhok, N.S., Ganem, N.J., Azioune, A., Thery, M., and Pellman, D. 2008. Mechanisms to suppress multipolar divisions in cancer cells with extra centrosomes. Genes \& Dev. 22: 2189-2203. 
Lingle, W.L., Barrett, S.L., Negron, V.C., D'Assoro, A.B., Boeneman, K., Liu, W., Whitehead, C.M., Reynolds, C., and Salisbury, J.L. 2002. Centrosome amplification drives chromosomal instability in breast tumor development. Proc. Nat1. Acad. Sci. 99: 1978-1983.

Margolis, B. and Borg, J.P. 2005. Apicobasal polarity complexes. J. Cell Sci. 118: 5157-5159.

Mitchison, T.J. 1992. Actin based motility on retraction fibers in mitotic PtK2 cells. Cell Motil. Cytoskeleton 22: 135-151.

Murphy, T.D. 2003. Drosophila skpA, a component of SCF ubiquitin ligases, regulates centrosome duplication independently of cyclin E accumulation. J. Cell Sci. 116: 2321-2332.

Musacchio, A. and Salmon, E.D. 2007. The spindle-assembly checkpoint in space and time. Nat. Rev. Mol. Cell Biol. 8: 379-393.

Nigg, E.A. 2007. Centrosome duplication: Of rules and licenses. Trends Cell Biol. 17: 215-221.

Quintyne, N.J., Reing, J.E., Hoffelder, D.R., Gollin, S.M., and Saunders, W.S. 2005. Spindle multipolarity is prevented by centrosomal clustering. Science 307: 127-129.

Raff, J.W. 2002. Centrosomes and cancer: Lessons from a TACC. Trends Cell Biol. 12: 222-225.

Ring, D., Hubble, R., and Kirschner, M. 1982. Mitosis in a cell with multiple centrioles. J. Cell Biol. 94: 549-556.

Saunders, W. 2005. Centrosomal amplification and spindle multipolarity in cancer cells. Semin. Cancer Biol. 15: 25-32.

Shi, X., Ma, Y.Q., Tu, Y., Chen, K., Wu, S., Fukuda, K., Qin, J., Plow, E.F., and Wu, C. 2007. The MIG-2/integrin interaction strengthens cell-matrix adhesion and modulates cell motility. J. Biol. Chem. 282: 20455-20466.

Shulman, J.M., Benton, R., and St Johnston, D. 2000. The Drosophila homolog of C. elegans PAR-1 organizes the oocyte cytoskeleton and directs oskar mRNA localization to the posterior pole. Cell 101: 377-388.

Skold, H.N., Komma, D.J., and Endow, S.A. 2005. Assembly pathway of the anastral Drosophila oocyte meiosis I spindle. J. Cell Sci. 118: 1745-1755.

Sotillo, R., Hernando, E., Diaz-Rodriguez, E., Teruya-Feldstein, J., Cordon-Cardo, C., Lowe, S.W., and Benezra, R. 2007. Mad2 overexpression promotes aneuploidy and tumorigenesis in mice. Cancer Cell 11: 9-23.

Tepass, U., Theres, C., and Knust, E. 1990. crumbs encodes an EGF-like protein expressed on apical membranes of Drosophila epithelial cells and required for organization of epithelia. Cell 61: 787-799.

Thery, M. and Bornens, M. 2006. Cell shape and cell division. Curr. Opin. Cell Biol. 18: 648-657.

Thery, M., Racine, V., Pepin, A., Piel, M., Chen, Y., Sibarita, J.B., and Bornens, M. 2005. The extracellular matrix guides the orientation of the cell division axis. Nat. Cell Biol. 7: 947953.

Thery, M., Jimenez-Dalmaroni, A., Racine, V., Bornens, M., and Julicher, F. 2007. Experimental and theoretical study of mitotic spindle orientation. Nature 447: 493-496.

Toyoshima, F. and Nishida, E. 2007. Integrin-mediated adhesion orients the spindle parallel to the substratum in an EB1and myosin X-dependent manner. EMBO J. 26: 1487-1498.

Tsou, M.F. and Stearns, T. 2006. Mechanism limiting centrosome duplication to once per cell cycle. Nature 442: 947951.

Tu, Y., Wu, S., Shi, X., Chen, K., and Wu, C. 2003. Migfilin and Mig-2 link focal adhesions to filamin and the actin cytoskeleton and function in cell shape modulation. Cell 113: 37-47.

Vaughan, K.T. 2005. TIP maker and TIP marker; EB1 as a master controller of microtubule plus ends. J. Cell Biol. 171: 197200.
Weaver, B.A., Silk, A.D., Montagna, C., Verdier-Pinard, P., and Cleveland, D.W. 2007. Aneuploidy acts both oncogenically and as a tumor suppressor. Cancer Cell 11: 25-36.

Yang, Z., Loncarek, J., Khodjakov, A., and Rieder, C.L. 2008. Extra centrosomes and/or chromosomes prolong mitosis in human cells. Nat. Cell Biol. 10: 748-751. 


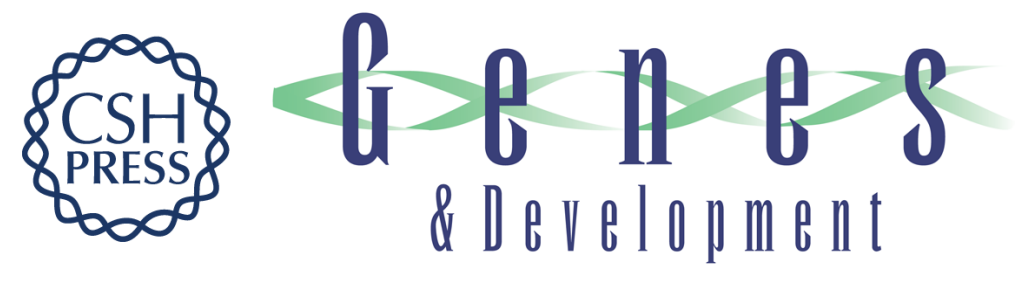

\section{Multiple centrosomes: together they stand, divided they fall}

Fanni Gergely and Renata Basto

Genes Dev. 2008, 22:

Access the most recent version at doi:10.1101/gad.1715208

Related Content Mechanisms to suppress multipolar divisions in cancer cells with extra centrosomes

Mijung Kwon, Susana A. Godinho, Namrata S. Chandhok, et al.

Genes Dev. August, 2008 22: 2189-2203

References This article cites 47 articles, 19 of which can be accessed free at:

http://genesdev.cshlp.org/content/22/17/2291.full.html\#ref-list-1

Articles cited in:

http://genesdev.cshlp.org/content/22/17/2291.full.html\#related-urls

\section{License}

Email Alerting

Receive free email alerts when new articles cite this article - sign up in the box at the top

Service right corner of the article or click here.

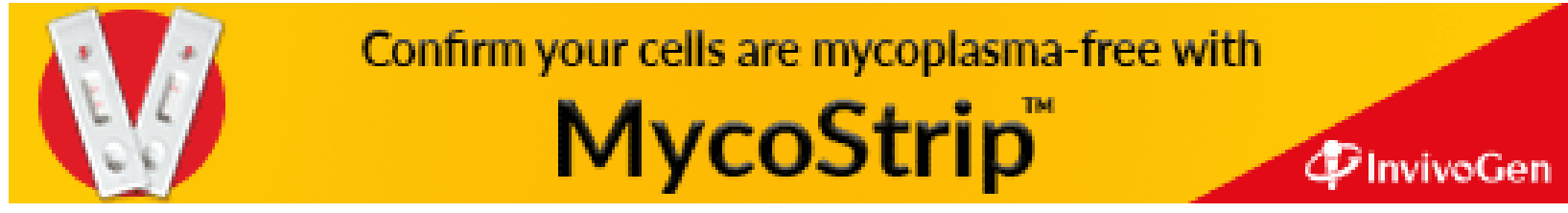

